\title{
Universal approximations for flavor models
}

\author{
Gero von Gersdorff \\ Physics Department, Pontifícia Universidade Católica, \\ Rua Marquês de São Vicente 225, Rio de Janeiro, Brazil \\ E-mail: gersdorff@gmail.com
}

ABSTRACT: We develop a systematic analytical approximation scheme for the singular value decompositions of arbitrary complex three dimensional matrices $Y$ with non-degenerate singular values. We derive exact expressions for the errors of this approximation and show that they are bounded from above by very simple ratios of the form $\left(y_{i} / y_{j}\right)^{2 n}$ where $y_{i}<y_{j}$ are singular values of $Y$ and $n$ is the order of the approximation. The applications we have in mind are the analytical and numerical treatments of arbitrary theories of flavor. We also compute upper bounds for the errors of the Cabbibo Kobayashi Maskawa (CKM) matrix that only depend on the ratios of the masses and the physical CKM angles.

KeYwords: Beyond Standard Model, Quark Masses and SM Parameters

ARXIV EPRINT: 1903.11077 


\section{Contents}

1 Introduction 1

2 Preliminaries 2

3 Approximate diagonalizations 3

4 Examples $\quad 8$

4.1 Frogatt-Nielsen and similar 8

4.2 Textures from spontaneously broken SU(2) symmetry 9

$\begin{array}{lll}4.3 & \text { Clockwork model } & 11\end{array}$

5 Conclusions 13

$\begin{array}{ll}\text { A Error analysis } & 13\end{array}$

\section{Introduction}

One of the unresolved mysteries of the Standard Model (SM) is the peculiar structure of the fermion sector, in particular the very non-generic structure of their masses and mixings. Disregarding the neutrino sector, the properties of the fermions are encoded in their Yukawa couplings, complex three by three matrices $Y_{u}, Y_{d}$ and $Y_{e}$.

A brief summary of the SM fermion data is given in table 1 . The actual values depend on the renormalization group scale (and scheme) as well as on possible New Physics thresholds (such as supersymmetry), we quote the SM values in the $\overline{\mathrm{MS}}$ scheme at $1 \mathrm{TeV}$ as given in ref. [1]. Notice that RG running and threshold corrections typically give only $\mathcal{O}(1)$ modifications to these numbers. One observes the hierarchical structure

$$
y_{u_{1}} \ll y_{u_{2}} \ll y_{u_{3}}, \quad y_{d_{1}} \ll y_{d_{2}} \ll y_{d_{3}}, \quad y_{e_{1}} \ll y_{e_{2}} \ll y_{e_{3}} .
$$

Moreover, the CKM mixing angles follow the hierarchy

$$
\theta_{13} \ll \theta_{23} \ll \theta_{12} \ll 1 .
$$

while mixing angles in the neutrino sector are $\mathcal{O}(1)$.

The observations eq. (1.1) require that the eigenvalues of the Hermitian matrices $Y_{x} Y_{x}^{\dagger}$ $(x=u, d, e)$ are very hierarchical, while eq. (1.2) implies that the eigenvectors of $Y_{u} Y_{u}^{\dagger}$ and $Y_{d} Y_{d}^{\dagger}$ are closely aligned. Clearly, the latter fact means that the up and down Yukawa couplings have to "know of each other" in some way.

Many models have been proposed to explain this peculiar structure, we comment on a few representative ones in section 4 . In order to have good analytical and numerical 


\begin{tabular}{|cccccc|cccc|}
\hline$y_{u}$ & $6.3 \times 10^{-6}$ & $y_{d}$ & $1.4 \times 10^{-5}$ & $\theta_{12}$ & 0.23 & $y_{e}$ & $2.8 \times 10^{-6}$ & $\theta_{12}$ & 0.58 \\
$y_{c}$ & $3.1 \times 10^{-3}$ & $y_{s}$ & $2.7 \times 10^{-4}$ & $\theta_{23}$ & $4.2 \times 10^{-2}$ & $y_{\mu}$ & $6.0 \times 10^{-4}$ & $\theta_{23}$ & 0.82 \\
$y_{t}$ & 0.87 & $y_{b}$ & $1.4 \times 10^{-2}$ & $\theta_{13}$ & $3.7 \times 10^{-3}$ & $y_{\tau}$ & $1.0 \times 10^{-2}$ & $\theta_{13}$ & 0.15 \\
\hline
\end{tabular}

Table 1. Quark and Lepton data at $1 \mathrm{TeV}$ in the SM [1].

control over the model parameter space, it is common practice to expand eigenvalues and eigenvectors in terms of some small parameters present in a given model. However the goodness of such expansions not only depends on the size of the expansion parameters (of which there might be several) but also on all the other parameters of the model. To judge its accuracy, one would have to go higher order in the expansion, and in order to achieve a given precision one has to resum the expansion up to a certain order. In this short paper we comment on a very powerful and fully model-independent approximation scheme that can be carried to arbitrarily high precision. It is extremely simple to apply and does not rely on the existence of any expansion parameter. The expressions for eigenvalues and mixings are given exclusively in terms of the $\left(Y_{x}\right)_{i j}$, without any assumptions on their sizes. The errors of the approximation are exactly bounded by (not just of the order of) simple ratios of the quantities $\left(y_{i} / y_{j}\right)^{2 n}, y_{i}<y_{j}$, where $n$ is the order of the approximation. We will see that known approximations of particular models follow without any calculation, with the added bonus of adding complete analytical control over their errors.

We hope that the method presented in this paper can help to better understand the parameter space of existing and yet to be conceived models of flavor, facilitating for instance fits to the data. Finally this paper may serve as a reference for students studying mainstream models (such as Frogatt-Nielsen [2]) for the first time who want a simple and easy to follow derivation of the known approximations.

\section{Preliminaries}

We recall that an arbitrary complex matrix $Y$ can be written as a so-called singular value decomposition (SVD)

$$
Y=U_{L} \mathcal{Y} U_{R}^{\dagger},
$$

where $U_{L}$ and $U_{R}$ are unitary and $\mathcal{Y}$ is diagonal. The elements of $\mathcal{Y}$ are called singular values (SV) of $Y$, which are unique up to phases. Given a particular phase convention for $\mathcal{Y}$ (say all SVs real positive), the remaining ambiguity consists of multiplication of $U_{L}$ and $U_{R}$ with the same diagonal phases matrix from the right. The columns of $U_{L}\left(U_{R}\right)$ are eigenvectors of $Y Y^{\dagger}\left(Y^{\dagger} Y\right)$, and $|\mathcal{Y}|^{2}$ are the eigenvalues.

Our discussion will be greatly simplified by a convenient parametrization of a general unitary matrix $U \in \mathrm{U}(3)$ which we write as

$$
U=K(a, b, c)\left(\begin{array}{ccc}
e^{i \alpha} & & \\
& e^{i \beta} & \\
& & e^{i \gamma}
\end{array}\right)
$$


with $K$ defined as

$$
K(a, b, c) \equiv\left(\begin{array}{ccc}
\frac{1}{n_{1}} & \frac{a-c^{*}(b-a c)}{n_{1} n_{3}} & \frac{b}{n_{3}} \\
\frac{-a^{*}}{n_{1}} & \frac{1+b^{*}(b-a c)}{n_{1} n_{3}} & \frac{c}{n_{3}} \\
\frac{-(b-a c)^{*}}{n_{1}} & \frac{-c^{*}-a b^{*}}{n_{1} n_{3}} & \frac{1}{n_{3}}
\end{array}\right) .
$$

We will refer to this parametrization as the $a b c$ parametrization. The quantities $n_{1}$ and $n_{3}$ are normalization constants for the column vectors, given by

$$
n_{1} \equiv \sqrt{1+|a|^{2}+|b-a c|^{2}}, \quad n_{3} \equiv \sqrt{1+|b|^{2}+|c|^{2}} .
$$

Notice that $\operatorname{det} K=1$. The numbers $a, b, c$ are arbitrary complex numbers, which together with the phases $\alpha, \beta, \gamma$ comprise the 9 (real) degrees of freedom of $\mathrm{U}(3)$. We will sometimes use the shorthand

$$
\tilde{b} \equiv b-a c .
$$

Given an arbitrary $U \in \mathrm{U}(3)$, one can readily compute these parameters:

$$
a=-\frac{U_{21}^{*}}{U_{11}^{*}}, \quad \tilde{b}=-\frac{U_{31}^{*}}{U_{11}^{*}}, \quad b=\frac{U_{13}}{U_{33}}, \quad c=\frac{U_{23}}{U_{33}} .
$$

and

$$
\alpha=\arg U_{11}, \quad \gamma=\arg U_{33}, \quad \alpha+\beta+\gamma=\arg \operatorname{det} U .
$$

Unitary matrices that appear in SVDs of random matrices (with matrix elements drawn from a uniform measure) are distributed according to the invariant Haar measure [3]. In the parametrization eq. (2.2), the Haar measure of the group is simply

$$
d U=\frac{1}{32 \pi^{6}} \frac{1}{n_{1}^{4} n_{3}^{4}} d a d a^{*} d b d b^{*} d c d c^{*} d \alpha d \beta d \gamma,
$$

where we normalized the measure to unity.

\section{Approximate diagonalizations}

In this section we are going to describe a neat analytic way of diagonalizing a Hermitian matrix. Even though in principle it can be applied to any matrix, it is particularly suited for matrices that have hierarchical spectra, such as the mass matrices of the SM.

Let $A$ be a positive-definite, Hermitian 3 by 3 matrix, and let $a_{i}$ be its (non-degenerate) eigenvalues. By convention, we will assume that $a_{1}<a_{2}<a_{3}$.

Let us consider the matrix $A^{n}$ which of course has the same eigenvectors as $A$. Diagonalizing it as

$$
\left(A^{n}\right)_{i j}=a_{k}^{n} U_{i k} U_{j k}^{*}
$$


we write the three column vectors of $A^{n}$ suggestively as

$$
\begin{aligned}
& \left(A^{n}\right)_{i 1}=a_{3}^{n} U_{13}^{*}\left(U_{i 3}+\left[\frac{a_{2}^{n}}{a_{3}^{n}} \frac{U_{12}^{*}}{U_{13}^{*}}\right] U_{i 2}+\left[\frac{a_{1}^{n}}{a_{3}^{n}} \frac{U_{11}^{*}}{U_{13}^{*}}\right] U_{i 1}\right), \\
& \left(A^{n}\right)_{i 2}=a_{3}^{n} U_{23}^{*}\left(U_{i 3}+\left[\frac{a_{2}^{n}}{a_{3}^{n}} \frac{U_{22}^{*}}{U_{23}^{*}}\right] U_{i 2}+\left[\frac{a_{1}^{n}}{a_{3}^{n}} \frac{U_{21}^{*}}{U_{23}^{*}}\right] U_{i 1}\right), \\
& \left(A^{n}\right)_{i 3}=a_{3}^{n} U_{33}^{*}\left(U_{i 3}+\left[\frac{a_{2}^{n}}{a_{3}^{n}} \frac{U_{32}^{*}}{U_{33}^{*}}\right] U_{i 2}+\left[\frac{a_{1}^{n}}{a_{3}^{n}} \frac{U_{31}^{*}}{U_{33}^{*}}\right] U_{i 1}\right) .
\end{aligned}
$$

Observe that we have written each column of $A^{n}$ as a linear combination of the eigenvectors of $A$. This way it becomes clear that as $n \rightarrow \infty$ each of the three column vectors of $A^{n}$ (when properly normalized) converges to $U_{i 3}$, the eigenvector corresponding to the largest eigenvalue $a_{3}$, and hence we can compute the $b$ and $c$ parameters of $U=K(a, b, c)$ as

$$
b=\lim _{n \rightarrow \infty} \frac{\left(A^{n}\right)_{1 j}}{\left(A^{n}\right)_{3 j}}, \quad c=\lim _{n \rightarrow \infty} \frac{\left(A^{n}\right)_{2 j}}{\left(A^{n}\right)_{3 j}} .
$$

We can choose any of the three representations $j=1,2,3$, but observe that for the $j$ th column of $A^{n}$ a small $\left|U_{j 3}\right|$ will slow down the convergence (or even destroy it if $U_{j 3}=0$ ) because it appears in the denominator of the subleading terms. However the $U_{j 3}$ cannot all be small simultaneously, in fact unitarity implies $\max _{j}\left|U_{j 3}\right| \geq \frac{1}{\sqrt{3}}$. Fortunately it is possible to find the $j$ that maximizes $\left|U_{j 3}\right|$ without actually knowing $U$, just by looking at the length of the $j^{\text {th }}$ column vector of $A^{n}$

$$
L_{j}^{2} \equiv \sum_{i}\left|\left(A^{n}\right)_{i j}\right|^{2}=\left|U_{j 3}\right|^{2} a_{3}^{2 n}+\left|U_{j 2}\right|^{2} a_{2}^{2 n}+\left|U_{j 1}\right|^{2} a_{1}^{2 n} .
$$

It is easy to see that in the limit of $n \rightarrow \infty$ the largest $\left|U_{j 3}\right|$ is exactly correlated with the largest $L_{j}$. For finite $n$ one can still find a lower bound for $\left|U_{j 3}\right|$ which is evaluated in appendix A and comes very close to $\frac{1}{\sqrt{3}}$. In order to achieve the smallest error, we thus choose the longest of the three column vectors of $A^{n}$, i.e., the one with the largest $L$, as an approximation for the eigenvector to eigenvalue $a_{3}$.

For the parameter $a$ and $\tilde{b}$ which are related to the smallest eigenvalue, we proceed with the same method, this time with the matrix $A^{-n}$,

$$
a^{*}=-\lim _{n \rightarrow \infty} \frac{\left(A^{-n}\right)_{2 k}}{\left(A^{-n}\right)_{1 k}}, \quad \tilde{b}^{*}=-\lim _{n \rightarrow \infty} \frac{\left(A^{-n}\right)_{3 k}}{\left(A^{-n}\right)_{1 k}},
$$

where for completeness we also give the dependent quantity $\tilde{b}$. Notice that if the columns used to determine $U_{i 3}$ and $U_{i 1}$ are different, $j \neq k$, the eigenvectors are exactly orthogonal (this trivially follows from the fact that $A^{-n}$ and $A^{n}$ are inverses) and equivalently $\tilde{b}=$ $b-a c$. On the contrary, it is possible that the maximum length criterion gives the same column (say the first one of both $A^{n}$ and $A^{-n}$ ), in this case they are only orthogonal up to small corrections (see appendix A for details). Sometimes it might be more convenient to work with the matrix of minors

$$
\tilde{A} \equiv \operatorname{det}(A)\left(A^{-1}\right)^{T},
$$


in which case one has

$$
a=-\lim _{n \rightarrow \infty} \frac{\left(\tilde{A}^{n}\right)_{2 k}}{\left(\tilde{A}^{n}\right)_{1 k}}, \quad \tilde{b}=-\lim _{n \rightarrow \infty} \frac{\left(\tilde{A}^{n}\right)_{3 k}}{\left(\tilde{A}^{n}\right)_{1 k}} .
$$

This way, the procedure is defined also for $a_{1}=0$.

Obviously, these results hold for matrices of any dimensions $d$, but the advantage for the case of interest of $d=3$ is that it already completely determines the full unitary matrix $U$, as the eigenvector to $a_{2}$ follows from

$$
U_{i 2}^{(n)}=\epsilon_{i \ell m} U_{\ell 3}^{*(n)} U_{m 1}^{*(n)},
$$

or, equivalently, from the $a b c$ representation in eq. (2.3).

Having found an approximation $U^{(n)}$ for the matrix $U$, our next goal is to find an upper bound for the error of our algorithm. To this end, let us define the matrix

$$
V \equiv U^{\dagger} U^{(n)}
$$

which must converge to the identity. An explicit expression for $V$ in terms of $U_{i j}$ and the $a_{i}$ can easily be obtained and is given in appendix $\mathrm{A}$, where it is also shown that $V$ can be bounded by

$$
\left(\begin{array}{ccc}
1-\left|V_{11}\right|^{2} & \left|V_{12}\right|^{2} & \left|V_{13}\right|^{2} \\
\left|V_{21}\right|^{2} & 1-\left|V_{22}\right|^{2} & \left|V_{23}\right|^{2} \\
\left|V_{31}\right|^{2} & \left|V_{32}\right|^{2} & 1-\left|V_{33}\right|^{2}
\end{array}\right) \leq 2\left(\begin{array}{lll}
\left(\frac{a_{1}}{a_{2}}\right)^{2 n} & \left(\frac{a_{1}}{a_{2}}\right)^{2 n} & \left(\frac{a_{1}}{a_{3}}\right)^{2 n} \\
\left(\frac{a_{1}}{a_{2}}\right)^{2 n} & \left(\frac{a_{1}}{a_{2}}\right)^{2 n}+\left(\frac{a_{2}}{a_{3}}\right)^{2 n} & \left(\frac{a_{2}}{a_{3}}\right)^{2 n} \\
\left(\frac{a_{1}}{a_{3}}\right)^{2 n} & \left(\frac{a_{2}}{a_{3}}\right)^{2 n} & \left(\frac{a_{2}}{a_{3}}\right)^{2 n}
\end{array}\right)
$$

Eq. (3.12) is valid for $j \neq k$ (which is commonly satisfied in many explicit models) while for the case $j=k$ a modified prescription yields the estimate eq. (A.18).

We summarize our findings in the following theorem.

Theorem 1 The longest column vector of the matrix $A^{n}\left(A^{-n}\right)$ converges to the eigenvector to the largest (smallest) eigenvalue of $A$. The errors are encoded in the matrix $V$ defined in eqs. (3.11) and can be bounded by eqs. (3.12) or (A.18) respectively.

This procedure works extremely well and can provide us with very useful analytic expressions and even fast converging numerical ones without the need for the calculation of the eigenvalues. ${ }^{1}$ Note that the matrix $U^{(n)}$ defines a converging sequence (whose elements are explicitely given in terms of the matrix elements $A_{i j}$ ). Convergence is guaranteed as long as the eigenvalues are only slightly different, even though convergence is of course faster for smaller ratios of the eigenvalues. It is remarkable that contrary to what eq. (3.12) might suggest, the resulting matrix $\lim _{n \rightarrow \infty} U^{(n)}$ is actually correct even when the spectra are degenerate. To see this, notice that the matrix $U$ is not unique in this case. For instance, when $a_{1} \neq a_{2}=a_{3}$ only the first eigenvector is fixed while the other two can

\footnotetext{
${ }^{1}$ Notice that for high-precision numerical calculations one can conveniently work with $n=2^{k}$ which only requires $k \sim \log n$ matrix products instead of $n$.
} 


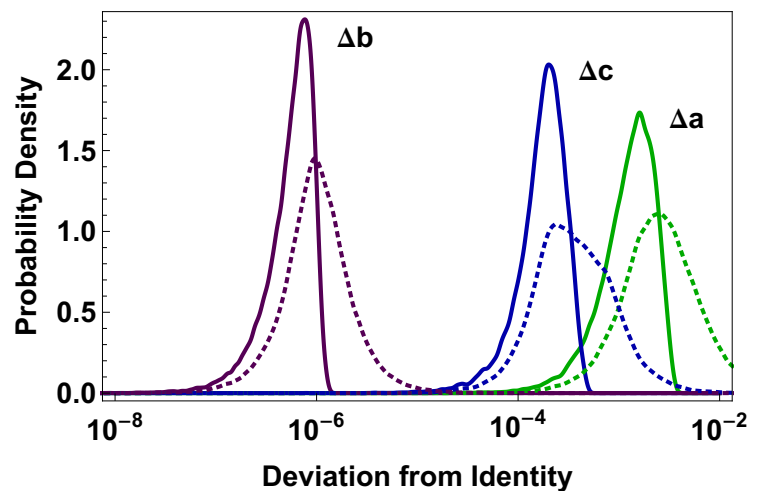

Figure 1. Left: error of the approximate eigenvectors for down-type Yukawa couplings in arbitrary bases (see text for details). The dotted lines are the corresponding approximations without the maximum length criterion (instead choosing say $j=1$ always), showing considerably worse errors.

be any vector orthogonal to $U_{i 1}$. In this particular case, the matrix $V$ in eq. (3.12) is convergent only in its first row and first vector $\left(\left|V_{i 1}\right| \rightarrow \delta_{i 1},\left|V_{1 i}\right| \rightarrow \delta_{1 i}\right)$, showing that the method is finding the correct eigenvector to $a_{1}$. The non-convergent two by two lower block of $V$ indicates that the remaining two eigenvectors are not aligned between $U$ and $U^{(n)}$. However, these eigenvectors are arbitrary and hence any unitary $U$ that has as its first column the eigenvector to $a_{1}$ is a correct solution. Similar considerations apply for $a_{1}=a_{2} \neq a_{3}$ as well as the trivial case $a_{1}=a_{2}=a_{3}$.

To illustrate the importance of the maximum-length criterion, let us numerically examine the error of our method for a hierarchical matrix with and without the maximum-length criterion. For definiteness, we consider $A=Y_{d} Y_{d}^{\dagger}$ with $Y_{d}$ the down quark Yukawa couplings (see table 1) in an arbitrary basis. Starting with the diagonal matrix, we perform random $\mathcal{O}(1)$ rotations $U$ (drawn from the distribution eq. (2.8)) and then recompute the matrix $U \approx U^{(n)}$ using our method with $n=1$. To measure the error, we compute the matrix $V \equiv U^{\dagger} U^{(n)}$ and find its corresponding parameters $\Delta a, \Delta b$, and $\Delta c$ which indicate the deviation of $V$ from the identity and are plotted in figure 1. The errors are in accordance with the upper limits derived above, while dropping the maximum-length criterion results in considerably larger corrections.

The eigenvalues themselves can be conveniently obtained by computing the traces of $A^{n}$ and $A^{-n}$.

$$
a_{3}=\lim _{n \rightarrow \infty}\left(\operatorname{tr} A^{n}\right)^{\frac{1}{n}}, \quad a_{1}=\lim _{n \rightarrow \infty}\left(\operatorname{tr} A^{-n}\right)^{-\frac{1}{n}},
$$

with $a_{2}$ following from the determinant. Alternatively, one can compute the limits

$$
a_{3}=\lim _{n \rightarrow \infty} \frac{\left(A^{n+1}\right)_{j j}}{\left(A^{n}\right)_{j j}}, \quad a_{1}=\lim _{n \rightarrow \infty} \frac{\left(A^{-(n+1)}\right)_{k k}}{\left(A^{-n}\right)_{k k}},
$$

where $j$ and $k$ are determined from the maximum length criterion.

Even though this procedure works for any Hermitian matrix, it is particularly wellsuited for very hierarchical matrices with spectra $a_{1} \ll a_{2} \ll a_{3}$, such as the mass matrices of the SM. In these cases one obtains very good approximations from the above relations 
even with $n=1$. Notice that we do not need to assume a particular form for the matrices, as long as the spectrum is hierarchical. These approximations thus go beyond the ones usually quoted in the literature, such as those resulting from the particular structure in eq. (4.1), which can be obtained as special cases of the above method.

If $A$ is given as $A=Y Y^{\dagger}$, one can also obtain "half-integer" approximations, which can easily be found by starting from the SVD of $Y, Y Y^{\dagger} Y$, etc, instead of eq. (3.1). Of particular interest is the $n=\frac{1}{2}$ case, obtained by starting from the SVD of $Y$, and results in the following important special case

Theorem 2 ( $n=\frac{1}{2}$ approximation.) The singular value decomposition of a complex matrix $Y$ is approximately determined as follows. The columns $\left(U_{L}\right)_{i 3}$ and $\left(U_{L}\right)_{i 1}$ are proportional to the longest columns of the matrices $Y$ and $\left(Y^{-1}\right)^{\dagger}$ respectively, and the columns $\left(U_{R}\right)_{i 3}$ and $\left(U_{R}\right)_{i 1}$ are proportional to the longest columns of the matrices $Y^{\dagger}$ and $Y^{-1}$. The errors are then bounded by eqs. (3.12) or (A.18) with $\left(a_{i}\right)^{n} \rightarrow y_{i}$.

As before, instead of $Y^{-1}$ one might equivalently work with the matrix of minors $\tilde{Y}=$ $\operatorname{det} Y\left(Y^{-1}\right)^{T}$.

We will verify in section 4 that for the special structure eq. (4.1) the case $n=\frac{1}{2}$ immediately yields the known approximations. Some corrections (such as the ones reported in ref. [4]) can be recovered from the $n=1$ approximation. Finally we notice that there exist approximate expressions for the eigenvalues for half-integer $n$. In the case of $n=\frac{1}{2}$, they read

$$
a_{3}=\frac{\left(Y Y^{\dagger}\right)_{j j}\left(Y^{\dagger} Y\right)_{j^{\prime} j^{\prime}}}{\left|Y_{j j^{\prime}}\right|^{2}}, \quad a_{1}=\frac{\left(\tilde{Y} \tilde{Y}^{\dagger}\right)_{k k}\left(\tilde{Y} Y^{\dagger} \tilde{Y}\right)_{k^{\prime} k^{\prime}}}{\left|\tilde{Y}_{k k^{\prime}}\right|^{2}}
$$

where here $j\left(j^{\prime}\right)$ labels the longest row (column) of $Y$ and analogously for $\tilde{Y}$.

Let us now move to the CKM matrix $V_{\mathrm{CKM}} \equiv U_{u}^{\dagger} U_{d}$. The latter reads in our approximation:

$$
V_{\mathrm{CKM}}^{(n)}=\left(U_{u}^{(n)}\right)^{\dagger} U_{d}^{(n)}=V_{u}^{\dagger} V_{\mathrm{CKM}} V_{d} .
$$

From the last expression, by use of the triangle inequality and eq. (3.12), one can easily obtain upper bounds on the errors: ${ }^{2}$

$$
\begin{aligned}
& \left|\Delta V_{\mathrm{CKM}}\right|_{12} \leq \epsilon_{12}^{d}+\epsilon_{12}^{u}+\theta_{13} \epsilon_{23}^{d}, \\
& \left|\Delta V_{\mathrm{CKM}}\right|_{23} \leq \epsilon_{23}^{d}+\epsilon_{23}^{u}+\theta_{13} \epsilon_{12}^{u}, \\
& \left|\Delta V_{\mathrm{CKM}}\right|_{13} \leq \epsilon_{13}^{d}+\epsilon_{13}^{u}+\theta_{12} \epsilon_{23}^{d}+\theta_{23} \epsilon_{12}^{u}+\epsilon_{23}^{d}, \epsilon_{12}^{u}
\end{aligned}
$$

where

$$
\epsilon_{i j}^{u} \equiv \sqrt{2}\left(\frac{y_{u_{i}}}{y_{u_{j}}}\right)^{2 n_{u}}, \quad \epsilon_{i j}^{d} \equiv \sqrt{2}\left(\frac{y_{d_{i}}}{y_{d_{j}}}\right)^{2 n_{d}} .
$$

Using the explicit values given in table 1, we notice that for any model of Yukawa couplings, the approximation with $n_{d}=1$ and $n_{u}=\frac{1}{2}$ gives already excellent accuracy (at most $5 \%$ with the exception of $\theta_{23}$ that has a maximal error of $12 \%$, dominated by $\epsilon_{23}^{u}$ ). Observe that

\footnotetext{
${ }^{2}$ We only consider the case $j \neq k$ for simplicity. We have used $\left|\left(V_{\mathrm{CKM}}\right)_{i j}\right| \leq \theta_{i j}$ and for clarity omitted strictly subleading terms in the upper bounds.
} 
our approximation is very well-suited for numerical fits. Away from the physical values for the Yukawas it might lead to large errors (when for instance the true eigenvalues are not hierarchical), nevertheless near the $\chi^{2}$ minimum one can always fully trust it.

We stress once more that the upper bounds for the errors are very conservative, and can be much less in particular models. This typically happens when the entries in the matrix $\mathrm{Y}$ are itself hierarchical. One can get a good idea of this effect by computing $V$ approximately using the approximate result for $U$. The result is the matrix $V^{(n)}$, given in eq. (A.21).

Let us also comment on the PMNS matrix for neutrinos. If neutrinos are moderately hierarchical, one can obtain decent approximations for low $n$. This is only possible for the normal hierarchy, as in the inverted case one has $\frac{m_{1}}{m_{2}}>0.98$. On the other hand, in the normal ordering case, one has $0.17 \leq \frac{m_{2}}{m_{3}} \leq 1$ and $0 \leq \frac{m_{1}}{m_{2}} \leq 1$ depending on the size of $m_{1}$. One can achieve accuracy comparable with (or less than) the current experimental one at $n=1$ if $m_{1} \lesssim 10^{-3} \mathrm{eV}$.

\section{Examples}

In this section, we would like to illustrate our method in three particular models or classes of models.

\subsection{Frogatt-Nielsen and similar}

Let us first turn to the Froggatt-Nielsen Model [2]. Identical reasoning applies to models with similar structure as in eq. (4.1), such as extra dimensions [6, 7] or certain Clockwork models [8]. The structure of the Yukawa couplings is given as follows

$$
Y_{u}=\left(\begin{array}{ccc}
\epsilon^{q_{1}} & & \\
& \epsilon^{q_{2}} & \\
& & \epsilon^{q_{3}}
\end{array}\right) \hat{Y}_{u}\left(\begin{array}{ccc}
\epsilon^{u_{1}} & & \\
& \epsilon^{u_{2}} & \\
& & \epsilon^{u_{3}}
\end{array}\right), \quad Y_{d}=\left(\begin{array}{ccc}
\epsilon^{q_{1}} & & \\
& \epsilon^{q_{2}} & \\
& & \epsilon^{q_{3}}
\end{array}\right) \hat{Y}_{d}\left(\begin{array}{ccc}
\epsilon^{d_{1}} & & \\
& \epsilon^{d_{2}} & \\
& & \epsilon^{d_{3}}
\end{array}\right) .
$$

where $\hat{Y}_{u, d}$ are $\mathcal{O}(1)$ complex matrices and the $q_{i}, u_{i}$ and $d_{i}$ are the Froggatt-Nielsen charges of the doublet quarks, up-quarks, and down quarks respectively, taken to be positive, and $\epsilon$ is a moderately small order parameter. For definiteness, we will consider the charge assignments [9]

$$
q=(4,2,0), \quad u=(4,2,0), \quad d=(2,1,1),
$$

with $\epsilon \approx 0.2$. In the $n=\frac{1}{2}$ approximation, this selects the third row of $Y_{u}^{*}$ and the first row of $\tilde{Y}_{u}$ determine $U_{R}^{u}$

$$
\left(U_{R}^{u}\right)_{i 3} \propto Y_{3 i}^{u *}, \quad\left(U_{R}^{u}\right)_{i 1} \propto \tilde{Y}_{1 i}^{u},
$$

and the third row of $Y_{d}^{*}$ and the first row of $\tilde{Y}_{d}$ determine $U_{R}^{d}$ :

$$
\left(U_{R}^{d}\right)_{i 3} \propto Y_{3 i}^{d *}, \quad\left(U_{R}^{d}\right)_{i 1} \propto \tilde{Y}_{1 i}^{d} .
$$

Similarly, it selects the third column of $Y_{u}$ and the first column of $\tilde{Y}_{u}^{*}$ to determine $U_{L}^{u}$,

$$
\left(U_{L}^{u}\right)_{i 3} \propto Y_{i 3}^{u}, \quad\left(U_{L}^{u}\right)_{i 1} \propto \tilde{Y}_{i 1}^{u *} .
$$


For $U_{L}^{d}$ we have to choose the first column of $\tilde{Y}_{d}$, and either the second or third column of $Y_{d}$

$$
\left(U_{L}^{d}\right)_{i 3} \propto\left\{\begin{array}{l}
Y_{i 2}^{d}\left|\hat{Y}_{32}^{d}\right|>\left|\hat{Y}_{33}\right| \\
Y_{i 3}^{d}\left|\hat{Y}_{32}^{d}\right|<\left|\hat{Y}_{33}\right|
\end{array}, \quad\left(U_{L}^{u}\right)_{i 1} \propto \tilde{Y}_{i 1}^{u *} .\right.
$$

One sees that one can directly read off the eigenvectors from the Yukawa matrices and its inverses. For the determination of the third column of $U_{L}^{d}$, it is instructive to compare with the $n=1$ approximation, which is always given by the third column of $Y^{d} Y^{d \dagger}$, or

$$
\left(U_{L}^{d}\right)_{i 3} \propto \hat{Y}_{33}^{d *} Y_{i 3}^{d}+\hat{Y}_{32}^{d *} Y_{i 2}^{d}+\epsilon \hat{Y}_{31}^{d *} Y_{i 1}^{d},
$$

which is essentially a weighted average of the two $n=\frac{1}{2}$ cases, plus an $\epsilon$-suppressed admixture of the first column (which by itself is already numerically suppressed compared to the second and third). The results here coincide with the ones that one would obtain when making a careful (but rather lengthy) expansion in terms of $\epsilon$ (see for instance ref. [9]). However, no calculation is ever necessary to obtain them, one simply reads them off from our standard rules. Notice that $U_{L}^{u, d}$ and $U_{R}^{u}$ are almost diagonal while $U_{R}^{d}$ is almost blockdiagonal. This implies that the four error matrices $V_{L, R}^{u, d}$ are even more diagonal than the conservative upper bound in eq. (3.12).

\subsection{Textures from spontaneously broken SU(2) symmetry}

As a second example, we chose the particular texture

$$
Y_{13}=Y_{31}=Y_{11}=0, \quad Y_{12}=-Y_{21},
$$

which has been explored originally in [10, 11] (see ref. [5] for a variant taking into account more recent measurements of the CKM angles). We will implement this texture in the following completely general parametrization:

$$
Y=\left(\begin{array}{ccc}
0 & \eta \sqrt{y_{1} y_{2}} & 0 \\
-\eta \sqrt{y_{1} y_{2}} & \eta^{2}\left(\beta+\alpha^{2}\right) y_{2} & \alpha \rho^{-1} \sqrt{y_{2} y_{3}} \\
0 & \alpha \rho \sqrt{y_{2} y_{3}} & \eta^{-2} y_{3}
\end{array}\right)
$$

which gives the inverse

$$
\left(Y^{\dagger}\right)^{-1}=\frac{1}{y_{1} y_{2} y_{3}} \tilde{Y}^{*}=\left(\begin{array}{ccc}
\frac{\beta^{*}}{y_{1}} & \frac{1}{\eta} \frac{1}{\sqrt{y_{1} y_{2}}}-\eta \alpha \rho \frac{1}{\sqrt{y_{1} y_{3}}} \\
-\frac{1}{\eta} \frac{1}{\sqrt{y_{1} y_{2}}} & 0 & 0 \\
\eta \alpha \rho^{-1} \frac{1}{\sqrt{y_{1} y_{3}}} & 0 & \eta^{2} \frac{1}{y_{3}}
\end{array}\right) .
$$

Using redefinitions of the fermion fields, we have removed all the phases except one, chosen to be $\arg \beta$. We stress that this parametrization is exact and no approximations have been made so far. It depends on five real parameters, $\eta, \rho \alpha,|\beta|$, and $\arg \beta$. Notice that the determinant constraint $\operatorname{det} Y=y_{1} y_{2} y_{3}$ is already implemented, but the constraints from the traces

$$
\operatorname{tr} Y Y^{\dagger}=y_{3}^{2}+y_{2}^{2}+y_{1}^{2} \approx y_{3}^{2}, \quad \operatorname{tr}\left(Y Y^{\dagger}\right)^{-1}=y_{1}^{-2}+y_{2}^{-2}+y_{3}^{-2} \approx y_{1}^{-2},
$$


will lead to two more relations between the five parameters that we will work out below. ${ }^{3}$ However, one can already obtain various useful inequalities. First notice that the traces of $Y Y^{\dagger}$ and its inverse imply $\left|Y_{i j}\right| \leq y_{3}$ and $\left|\left(Y^{-1}\right)_{i j}\right| \leq y_{1}^{-1}$, in particular one has

$$
\begin{array}{rlrl}
1 & \leq \eta \leq \sqrt{\frac{y_{3}}{y_{1}}} \\
|\beta| \leq 1, & \left|\alpha^{2}+\beta\right| & \leq \frac{1}{\eta^{2}} \frac{y_{3}}{y_{2}} \\
\alpha \rho^{ \pm 1} \leq \sqrt{\frac{y_{3}}{y_{2}}}, & \alpha \rho^{ \pm 1} & \leq \frac{1}{\eta} \sqrt{\frac{y_{3}}{y_{1}}}
\end{array}
$$

from which one can for instance see that $\left|Y_{21}\right|^{2},\left|Y_{12}\right|^{2},\left|\left(Y^{-1}\right)_{21}\right|^{2}$ and $\left|\left(Y^{-1}\right)_{12}\right|^{2}$ contribute only very little to the traces in eq. (4.11).

The parameter $\eta$ interpolates between various qualitatively different regimes. We will be focusing on the lower end of the interval eq. (4.12), more precisely

$$
1 \leq \eta^{2} \ll \frac{y_{3}}{y_{1}},
$$

which provides the most interesting phenomenological models. Under this assumption one finds immediately that eqs. (4.11) reduce to

$$
\begin{aligned}
& |\beta|=1, \\
& \alpha^{2}=\left(\sqrt{\sinh ^{2} \xi+\eta^{4}}-\cosh \xi\right) \eta^{-4} \frac{y_{3}}{y_{2}} .
\end{aligned}
$$

Besides $\arg \beta$ this leaves as the only free parameters $\eta$ (constrained by eq. (4.15)) and $\xi$ or $\rho$ (unconstrained).

Let us now consider the matrix $U_{L}$. In all expressions below we leave the implementation of eq. (4.16) and (4.17) implicit, but in order to get a better feeling for the behavior of the results we show in figure 2 a plot of the relevant functions $\alpha$, and $\alpha \rho^{ \pm 1}$ as a function of $\xi$. Starting with the eigenvector to $y_{1}$, which is always given by the first column of $\tilde{Y}^{*}$ for the regime in eq. (4.15), one finds

$$
a=\frac{\beta^{*}}{\eta} \sqrt{\frac{y_{1}}{y_{2}}}, \quad \tilde{b}=-\frac{\eta \alpha \beta^{*}}{\rho} \sqrt{\frac{y_{1}}{y_{3}}} .
$$

Note that $|a|=\eta^{-1} \sqrt{\frac{y_{1}}{y_{2}}}$ and $|\tilde{b}| \leq \eta \sqrt{1-\eta^{-4}} \sqrt{\frac{y_{1}}{y_{2}}}$ (saturated at $\rho=0$ ). Moving on to the eigenvector to $y_{3}$, note that the first column of $Y$ can never be the longest, whereas the third one is the longest when $\left|Y_{33}\right|>\left|Y_{23}\right|$ or $\alpha \rho \eta^{2} \leq \sqrt{\frac{y_{3}}{y_{2}}}$. In this case $(j=3)$ one reads off

$$
b=0, \quad c=\frac{\alpha}{\rho} \eta^{2} \sqrt{\frac{y_{2}}{y_{3}}} .
$$

\footnotetext{
${ }^{3}$ The original matrix, defined by the constraint eq. (4.8), had ten free parameters subject to four possible phase redefinitions. After implementing the determinant and trace constraints, we will essentially have traded three of the six irreducible parameters by the eigenvalues $y_{i}$, leaving over three free parameters, taken to be $\rho, \arg \beta$ and $\eta$.
} 


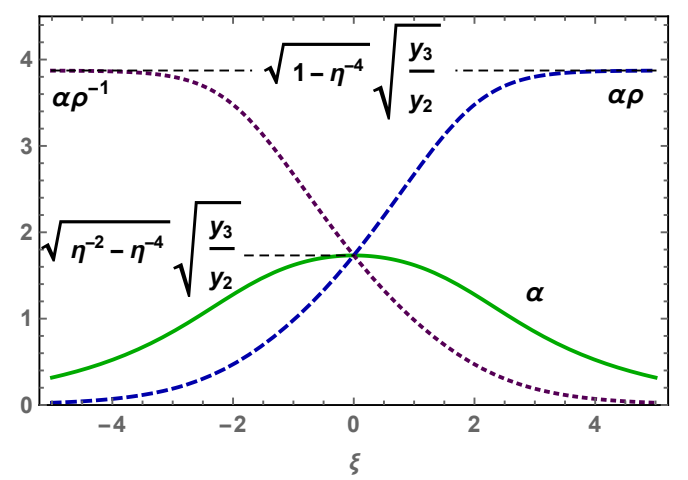

Figure 2. The functions $\alpha$ and $\alpha \rho^{ \pm 1}$ as a function of $\xi$.

There appears an upper bound for $c$ given by $|c| \leq \sqrt{\eta^{4}-1}$, saturated at $\rho=0$. Finally, when $\left|Y_{23}\right|>\left|Y_{33}\right|$ or $\alpha \rho \eta^{2} \geq \sqrt{\frac{y_{3}}{y_{2}}}$, the longest column of $Y$ is the second one. The criterion can only be satisfied for $\eta \geq 2^{\frac{1}{4}}$, which in turn means that $j=3$ is guaranteed when this condition is not met. For $j=2$, the $b$ and $c$ parameters read

$$
b=\frac{\eta}{\alpha \rho} \sqrt{\frac{y_{1}}{y_{3}}}, \quad c=\left(\frac{\alpha}{\rho}+\frac{\beta}{\alpha \rho}\right) \eta^{2} \sqrt{\frac{y_{2}}{y_{3}}} .
$$

Notice that $b$ is bounded by $|b| \leq \eta^{3} \frac{\sqrt{y_{1} y_{2}}}{y_{3}}$, which is small but still enhanced compared to the error of the approximation.

We see that as in the Froggatt Nielsen case, some of the rotation angles are suppressed. The global bounds (marginalized over $\rho$ ) are

$$
|a|^{2}=\frac{1}{\eta^{2}} \frac{y_{1}}{y_{2}}, \quad|\tilde{b}|^{2} \leq \frac{\eta^{4}-1}{\eta^{2}} \frac{y_{1}}{y_{2}}, \quad|b|^{2} \leq \eta^{6} \frac{y_{1} y_{2}}{y_{3}^{2}}, \quad|c|^{2} \leq \eta^{4}-1,
$$

which only depend on $\eta$. We notice that $c$ can only be suppressed if $\eta$ is close to one, which in particular implies $j=3$. We find it interesting that such strong statements as the ones in eq. (4.21) can easily be obtained within our formulation.

The matrix $U_{R}$ can be obtained by the interchange of $\rho \leftrightarrow \rho^{-1}$ in the above discussion.

To conclude, we stress again that the parametrization chosen here only depends on three free parameters $\rho, \eta, \arg \beta$, all the while being completely general apart from the mild assumption eq. (4.15). This has to be contrasted with explicit models, where the Yukawa couplings are typically parametrized in terms of many more parameters, leading to plenty of flat directions when performing fits to the masses and mixings. We therefore hope that our parametrization together with the approximation scheme developed in this paper greatly simplifies the task of finding phenomenologically viable parameters for models with certain textures.

\subsection{Clockwork model}

As a third example we will consider a simple Clockwork [12] model similar to the ones of refs. $[13,14]$. This is an example of a model that does not feature any obvious small 
expansion parameter, nor do we expect the rotation angles to be small. This class of models thus nicely illustrates how neither of the two properties are required for our approximation to work. The Yukawa couplings are generated from the following Clockwork Lagrangian:

$$
\begin{aligned}
\mathcal{L}= & \sum_{i=1}^{N_{q}} \bar{q}_{i} \not p q_{i}-\left(\bar{q}_{R}^{i} M_{q} q_{L}^{i}+\bar{q}_{R}^{i} K_{q} q_{L}^{i-1}+\text { h.c. }\right) \\
& +\sum_{i=1}^{N_{u}} \bar{u}_{i} \not p u_{i}-\left(\bar{u}_{L}^{i} M_{u} u_{R}^{i}+\bar{u}_{L}^{i} K_{u} u_{R}^{i-1}+\text { h.c. }\right) \\
& +\sum_{i=1}^{N_{d}} \bar{d}_{i} \not p d_{i}-\left(\bar{d}_{L}^{i} M_{d} d_{R}^{i}+\bar{d}_{L}^{i} K_{d} d_{R}^{i-1}+\text { h.c. }\right) \\
& +\bar{q}_{L}^{0} \not p q_{L}^{0}+\bar{u}_{R}^{0} \not p u_{R}^{0}+\bar{d}_{R}^{0} \not p d_{R}^{0}-\left(\bar{q}_{L}^{0} H d_{R}^{0}+\bar{d}_{L}^{0} \tilde{H} u_{R}^{0}+\text { h.c. }\right) .
\end{aligned}
$$

The last line has a $\mathrm{U}(3)$ flavor symmetry that is broken by the matrices $M$ and $K$. Integrating out the Clockwork fields one finds the effective Lagrangian

$$
\mathcal{L}_{\text {eff }}=\bar{q}_{L}^{0} Z_{q} \not p q_{L}^{0}+\bar{u}_{R}^{0} Z_{u} \not p u_{R}^{0}+\bar{d}_{R}^{0} Z_{d} \not p d_{R}^{0}-\left(\bar{q}_{L}^{0} H d_{R}^{0}+\bar{d}_{L}^{0} \tilde{H} u_{R}^{0}+\text { h.c. }\right),
$$

where

$$
Z_{x}=\sum_{k=0}^{N_{x}}\left(Q_{x}^{\dagger}\right)^{k}\left(Q_{x}\right)^{k}, \quad Q_{x}=M_{x}^{-1} K_{x} .
$$

For $K$ and $M$ random order one matrices, the eigenvalues of the Hermitian matrices $Z_{x}$ are always greater than one and strongly hierarchical for large $N_{x}$ [13]. The physical Yukawa couplings can then be obtained by canonical normalization

$$
Y_{u}=Z_{q}^{-\frac{1}{2}} Z_{u}^{-\frac{1}{2}}, \quad Y_{d}=Z_{q}^{-\frac{1}{2}} Z_{d}^{-\frac{1}{2}} .
$$

The hierarchical structure of the CKM angles is guaranteed by the common hierarchical factor $Z_{q}^{-\frac{1}{2}}$.

We have simulated the Yukawa couplings of this model, using random complex matrices (with flat priors) for $M$ and $K$. We focus on one Yukawa, say $Y_{u}$ with $N_{u}=N_{q}=5$ for definiteness. We have calculated the matrices $U_{L}$ and $U_{R}$, using our approximation with $n=\frac{1}{2}$, as well as the error matrices $V_{L}$ and $V_{R}$. The distributions for the $\left.\left|\delta_{i j}-\right| V_{i j}\right|^{2} \mid$, normalized to their respective bounds from eq. (3.12), are shown in figure $3 .{ }^{4}$ These ratios are expected to be smaller than one for our bounds to be correct. Indeed, no points in our simulation violate the bounds, even though they can come arbitrarily close. The median of the distribution is approximately equal to 0.15. We have checked that the displayed distribution does not depend significantly neither on the order of the approximation nor on the size of the generated hierarchies. In conclusion, this example illustrates that, barring further information on the structure of the Yukawa couplings, the global error bounds for our approximation are optimal.

\footnotetext{
${ }^{4}$ We only show the results for $j \neq k$, valid in about $90 \%$ of the simulation. We use the exact expressions for $X$ and $Z$ given in eq. (A.10) and (A.12) in order to account for the occasional cases in which the eigenvalues are not too hierarchical. We have also removed cases in which the hierarchies become so mild that the bounds exceed one, which will trivially be satisfied by any unitary matrix.
} 


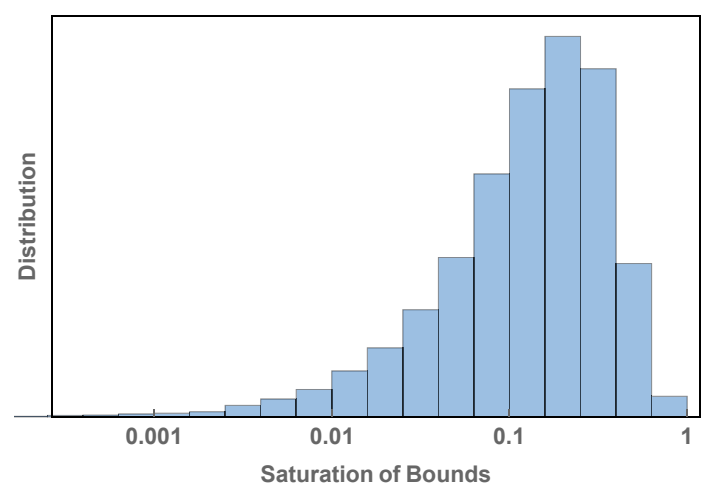

Figure 3. Distribution of the quantities $\left.\left|\delta_{i j}-\right| V_{i j}\right|^{2} \mid$ normalized to their respective bounds.

\section{Conclusions}

Finally let us summarize some of the features of the approximation developed here.

- It is universal, i.e., it does not make any assumptions about the underlying matrix $Y$.

- Rather than an expansion scheme (with typically unknown radius of convergence) in which one would need to compute higher and higher terms that need to be summed in order to achieve a desired accuracy, one directly obtains the result up to the desired accuracy in terms of the matrix elements of $Y$.

- We have derived a global upper bound for the error matrix $V$. Thus, there are no hidden cases where one looses control over the approximation. One can even obtain an approximate expression for $V$ by plugging in the leading result, see eq. (A.21).

- In contrast to usual expansion schemes, which contain often several small parameters, no ambiguities arise as to the relative size of various expansion parameters.

- We also have derived two equivalent but nontrivially related approximate expressions for the eigenvalues that can also be taken to arbitrary precision.

- The method is well-suited for numerical fits as it is very simple and computationally inexpensive. Moreover, even though the approximation might have large errors away from the physical parameters, in their vicinity (that is, near the " $\chi^{2}$ minimum") it is necessarily fully under control.

\section{A Error analysis}

In this section we analyze the error of our approximation. Let $U$ be the matrix whose columns are the eigenvectors of $A$, and let $a_{3}$ and $a_{1}$ be the largest and smallest eigenvalues respectively. Furthermore, let the $j$ th column of $A^{n}$ and the $k$ th column of $A^{-n}$ to be the longest. For now we will assume that $k \neq j$. Furthermore, let $U_{i 3}^{(n)}$ be the normalized $j$ th column of $A^{n}$ and $U_{i 1}^{(n)}$ be the normalized $k$ th column of $A^{-n}$, and let

$$
U_{i 2}^{(n)}=\epsilon_{i \ell m} U_{\ell 3}^{*(n)} U_{m 1}^{*(n)} .
$$


Since $j \neq k$, the matrix $U^{(n)}$ is unitary as $U_{i 1}^{(n)}$ and $U_{i 3}^{(n)}$ are exactly orthogonal in that case. Let us define the matrix

$$
V=U^{\dagger} U^{(n)}
$$

The matrix $V$ is unitary and converges to the identity, encoding the error of our approximation. It is easy to obtain explicitly its first and third columns, up to normalization:

$$
V_{i 1} \propto a_{i}^{-n} U_{k i}^{*}, \quad V_{i 3} \propto a_{i}^{n} U_{j i}^{*} .
$$

Note that orthogonality of these two columns is exact for $j \neq k$. In the $a b c$ representation $V$ can be given by $K(\Delta a, \Delta b, \Delta c)$ with

$$
\begin{aligned}
\Delta a & =-\frac{U_{k 2}}{U_{k 1}}\left(\frac{a_{1}}{a_{2}}\right)^{n}, & \Delta \tilde{b} & =-\frac{U_{k 3}}{U_{k 1}}\left(\frac{a_{1}}{a_{3}}\right)^{n}, \\
\Delta b & =\frac{U_{j 1}^{*}}{U_{j 3}^{*}}\left(\frac{a_{1}}{a_{3}}\right)^{n}, & \Delta c & =\frac{U_{j 2}^{*}}{U_{j 3}^{*}}\left(\frac{a_{2}}{a_{3}}\right)^{n} .
\end{aligned}
$$

These expressions are exact, but depend on the unknown matrix $U$. Owing to the longestcolumn criteria and the unitarity of the matrix $U$ the prefactors can be bounded as follows. Let $j$ maximize the column of $A^{n}$ and let $\ell \neq j$. Define $\xi_{\ell}$ to be the ratios of the lengths squared of the shorter columns to the longest, $\xi_{\ell} \equiv\left(A^{2 n}\right)_{\ell \ell} /\left(A^{2 n}\right)_{j j} \leq 1$, or

$$
\left|U_{j 3}\right|^{2} a_{3}^{2 n}+\left|U_{j 2}\right|^{2} a_{2}^{2 n}+\left|U_{j 1}\right|^{2} a_{1}^{2 n}=\xi_{\ell}^{-1}\left(\left|U_{\ell 3}\right|^{2} a_{3}^{2 n}+\left|U_{\ell 2}\right|^{2} a_{2}^{2 n}+\left|U_{\ell 1}\right|^{2} a_{1}^{2 n}\right) .
$$

We want to find a lower bound for $\left|U_{j 3}\right|$ as this quantity appears in the denominators of eq. (A.5). Maximizing the l.h.s. and minimizing the r.h.s. for fixed $U_{i 3}$ gives

$$
\left|U_{j 3}\right|^{2}\left(a_{3}^{2 n}-a_{2}^{2 n}\right)+a_{2}^{2 n} \geq \xi_{\ell}^{-1}\left(\left|U_{\ell 3}\right|^{2}\left(a_{3}^{2 n}-a_{1}^{2 n}\right)+a_{1}^{2 n}\right)
$$

(which by the way implies that $\xi_{\ell} \geq\left(\frac{a_{1}}{a_{3}}\right)^{2 n}$ ). We now use unitarity of the third column $U_{i 3}$ to find

$$
\left|U_{j 3}\right|^{2} \geq \frac{a_{3}^{2 n}-\xi a_{2}^{2 n}+a_{1}^{2 n}}{(1+\xi) a_{3}^{2 n}-\xi a_{2}^{2 n}+a_{1}^{2 n}}
$$

where $\xi \equiv \xi_{\ell}+\xi_{\ell^{\prime}}$, which is bounded by $2\left(\frac{a_{1}}{a_{3}}\right)^{2 n} \leq \xi \leq 2$. Finally use unitarity of the $j$ th row to get ${ }^{5}$

$$
\frac{\left|U_{j 1}\right|^{2}}{\left|U_{j 3}\right|^{2}}+\frac{\left|U_{j 2}\right|^{2}}{\left|U_{j 3}\right|^{2}} \leq \frac{\xi a_{3}^{2 n}-2 a_{1}^{2 n}}{a_{3}^{2 n}-\xi a_{2}^{2 n}+a_{1}^{2 n}} \equiv X_{\xi}
$$

The bound is the weakest at $\xi=2$ (when all three columns have equal lengths):

$$
X_{\xi} \leq 2 \frac{a_{3}^{2 n}-a_{1}^{2 n}}{a_{3}^{2 n}-2 a_{2}^{2 n}+a_{1}^{2 n}} \equiv X .
$$

Similar reasonings apply to the matrix $A^{-n}$, leading to

$$
\frac{\left|U_{k 3}\right|^{2}}{\left|U_{k 1}\right|^{2}}+\frac{\left|U_{k 2}\right|^{2}}{\left|U_{k 1}\right|^{2}} \leq \frac{\zeta a_{1}^{-2 n}-2 a_{3}^{-2 n}}{a_{1}^{-2 n}-\zeta a_{2}^{-2 n}+a_{3}^{-2 n}} \equiv Z_{\zeta},
$$

\footnotetext{
${ }^{5}$ Valid only for $a_{3}^{2 n}-\xi a_{2}^{2 n}+a_{1}^{2 n}>0$. We simply define $X_{\xi}$ to be infinite otherwise.
} 
where $\zeta$ is the analogous parameter for the inverse matrix, satisfying also $2\left(\frac{a_{1}}{a_{3}}\right)^{2 n} \leq \zeta \leq 2$. Again one has the global, $\zeta$-independent bound

$$
Z_{\zeta} \leq 2 \frac{a_{1}^{-2 n}-a_{3}^{-2 n}}{a_{1}^{-2 n}-2 a_{2}^{-2 n}+a_{3}^{-2 n}} \equiv Z .
$$

We can use these results to put upper bounds on the $a b c$ parameters of $V$ :

$$
\begin{array}{rlrl}
|\Delta a| & \leq \sqrt{Z}\left(\frac{a_{1}}{a_{2}}\right)^{n}, & |\Delta \tilde{b}| \leq \sqrt{Z}\left(\frac{a_{1}}{a_{3}}\right)^{n} \\
|\Delta b| \leq \sqrt{X}\left(\frac{a_{1}}{a_{3}}\right)^{n}, & |\Delta c| \leq \sqrt{X}\left(\frac{a_{2}}{a_{3}}\right)^{n} \\
|\Delta b|^{2}+|\Delta c|^{2} \leq X\left(\frac{a_{2}}{a_{3}}\right)^{2 n}, & |\Delta a|^{2}+|\Delta \tilde{b}|^{2} \leq Z\left(\frac{a_{1}}{a_{2}}\right)^{2 n} .
\end{array}
$$

These bounds are exact and only depend on the ratios of eigenvalues. Moreover, for large hierarchies or large $n$ we can very well approximate $X \approx 2, Z \approx 2$, which are precisely the bounds one would obtain if it were possible to choose $j$ to maximize $\left|U_{j 3}\right|$ directly. On the other hand, one can obtain stronger bounds $X_{\xi}<X$ and $Z_{\zeta}<Z$ if one takes the information on the length ratios into account.

It is now straightforward to put bounds on the matrix $V$ :

$$
\begin{aligned}
\left(\begin{array}{ccc}
1-\left|V_{11}\right|^{2} & \left|V_{12}\right|^{2} & \left|V_{13}\right|^{2} \\
\left|V_{21}\right|^{2} & 1-\left|V_{22}\right|^{2} & \left|V_{23}\right|^{2} \\
\left|V_{31}\right|^{2} & \left|V_{32}\right|^{2} & 1-\left|V_{33}\right|^{2}
\end{array}\right) & \leq\left(\begin{array}{ccc}
|\Delta a|^{2}+|\Delta \tilde{b}|^{2} & |\Delta a|^{2}+|\Delta \tilde{b}|^{2} & |\Delta b|^{2} \\
|\Delta a|^{2} & |\Delta a|^{2}+|\Delta c|^{2} & |\Delta c|^{2} \\
|\Delta \tilde{b}|^{2} & |\Delta b|^{2}+|\Delta c|^{2} & |\Delta b|^{2}+\left|\Delta c^{2}\right|
\end{array}\right) \\
& \leq\left(\begin{array}{ccc}
Z\left(\frac{a_{1}}{a_{2}}\right)^{2 n} & Z\left(\frac{a_{1}}{a_{2}}\right)^{2 n} & X\left(\frac{a_{1}}{a_{3}}\right)^{2 n} \\
Z\left(\frac{a_{1}}{a_{2}}\right)^{2 n} & Z\left(\frac{a_{1}}{a_{2}}\right)^{2 n}+X\left(\frac{a_{2}}{a_{3}}\right)^{2 n} & X\left(\frac{a_{2}}{a_{3}}\right)^{2 n} \\
Z\left(\frac{a_{1}}{a_{3}}\right)^{2 n} & X\left(\frac{a_{2}}{a_{3}}\right)^{2 n} & X\left(\frac{a_{2}}{a_{3}}\right)^{2 n}
\end{array}\right)
\end{aligned}
$$

where the first row is correct for any unitary matrix $K(\Delta a, \Delta b, \Delta c)$. These bounds are exact. They are optimized for small values of the ratios $\left(a_{i} / a_{j}\right)^{n}$, where $X \approx Z \approx 2$. As $V$ is unitary, the bounds become trivially satisfied when any of the entries on the r.h.s. of eq. (A.16) become greater than one. This roughly occurs when $\left(\frac{a_{2}}{a_{3}}\right)^{2 n}>\frac{1}{4}$ or $\left(\frac{a_{1}}{a_{2}}\right)^{2 n}>\frac{1}{4}$.

When it happens that $j=k$, one no longer has that $U_{i 1}^{(n)}$ and $U_{i 3}^{(n)}$ are exactly orthogonal. In practice, one can simply ignore this small deviation from unitarity (which can be estimated as $\left.U_{i 3}^{*(n)} U_{i 1}^{(n)} \leq 3\left(\frac{a_{1}}{a_{3}}\right)^{n}\right)$ but the error estimates are more complicated as $V$ is no longer unitary. Alternatively one can enforce unitarity by computing $\tilde{b}=b-a c$ instead of extracting it from $U_{i 1}^{(n)}$, however, this can introduce large errors in sufficiently pathological cases.

For the purpose of deriving robust upper bounds for the error, we follow a simpler approach and instead enforce $k \neq j$ (and hence exact unitarity of $U^{(n)}$ and $V$ ) by taking 
the second longest column of $A^{-n}{ }^{6}$ In this case one obtains a slightly weaker bound as follows. As $U_{j 3} \geq \sqrt{\frac{1}{3}}$, one has $U_{j 1} \leq \sqrt{\frac{2}{3}}$ and hence for the second largest entry one still has a lower bound, $\left|U_{k 1}\right| \geq \sqrt{\frac{1}{6}}$. Then, unitarity of the $k$ th row implies that $\left|U_{k 2}\right| \leq \sqrt{\frac{5}{6}}$ while unitarity of the third column implies $\left|U_{k 3}\right| \leq \sqrt{\frac{2}{3}}$. As a consequence, one has now that

$$
|\Delta a| \leq \sqrt{5}\left(\frac{a_{1}}{a_{2}}\right)^{n}, \quad|\Delta \tilde{b}| \leq 2\left(\frac{a_{1}}{a_{3}}\right)^{n}
$$

while eq. (A.14) still remains valid. In summary, the errors are now bounded by

$$
\left(\begin{array}{ccc}
1-\left|V_{11}\right|^{2} & \left|V_{12}\right|^{2} & \left|V_{13}\right|^{2} \\
\left|V_{21}\right|^{2} & 1-\left|V_{22}\right|^{2} & \left|V_{23}\right|^{2} \\
\left|V_{31}\right|^{2} & \left|V_{32}\right|^{2} & 1-\left|V_{33}\right|^{2}
\end{array}\right) \leq\left(\begin{array}{ccc}
5\left(\frac{a_{1}}{a_{2}}\right)^{2 n} & 5\left(\frac{a_{1}}{a_{2}}\right)^{2 n} & 2\left(\frac{a_{1}}{a_{3}}\right)^{2 n} \\
5\left(\frac{a_{1}}{a_{2}}\right)^{2 n} & 5\left(\frac{a_{1}}{a_{2}}\right)^{2 n}+2\left(\frac{a_{2}}{a_{3}}\right)^{2 n} & 2\left(\frac{a_{2}}{a_{3}}\right)^{2 n} \\
4\left(\frac{a_{1}}{a_{3}}\right)^{2 n} & 2\left(\frac{a_{2}}{a_{3}}\right)^{2 n} & 2\left(\frac{a_{2}}{a_{3}}\right)^{2 n}
\end{array}\right)
$$

It is clear that one could also work with the case of the longest column of $A^{-n}$ and the second longest column of $A^{n}$, but we will not spell out this case in detail.

For $n=\frac{1}{2}$, one replaces eq. (A.3) by

$$
\left(V_{L}\right)_{i 1} \propto y_{i}^{-1}\left(U_{R}\right)_{k i}^{*}, \quad\left(V_{L}\right)_{i 3} \propto y_{i}\left(U_{R}\right)_{j i}^{*}
$$

and

$$
\left(V_{R}\right)_{i 1} \propto y_{i}^{-1}\left(U_{L}\right)_{k i}^{*}, \quad\left(V_{R}\right)_{i 3} \propto y_{i}\left(U_{L}\right)_{j i}^{*} .
$$

The bounds one obtains are then given by setting $a_{i}=y_{i}^{2}$ and $n=\frac{1}{2}\left(\right.$ or $a_{i}^{n} \rightarrow y_{i}$ ) in the previous expressions.

It should be noted that the error matrix $V$ can itself be computed approximatively by replacing in eqs. (A.4) and (A.5) the $n$th order result for $U$. This can be useful as sometimes the quantities $\Delta a, \Delta b, \Delta c$ are more suppressed than the conservative upper bounds presented here, leading to much more accurate results. Calling this matrix $V^{(n)}$, it is given explicitly by

$$
V^{(n)} \approx\left(\begin{array}{ccc}
1 & -\frac{U_{k 2}^{(n)}}{U_{k 1}^{(n)}}\left(\frac{a_{1}}{a_{2}}\right)^{n} & \frac{U_{j 1}^{*(n)}}{U_{j 3}^{*(n)}}\left(\frac{a_{1}}{a_{3}}\right)^{n} \\
\frac{U_{k 2}^{*(n)}}{U_{k 1}^{*(n)}}\left(\frac{a_{1}}{a_{2}}\right)^{n} & 1 & \frac{U_{j 2}^{*(n)}}{U_{j 3}^{*(n)}}\left(\frac{a_{2}}{a_{3}}\right)^{n} \\
\frac{U_{k 3}^{*(n)}}{U_{k 1}^{*(n)}}\left(\frac{a_{1}}{a_{3}}\right)^{n} & -\frac{U_{j 2}^{(n)}}{U_{j 3}^{(n)}}\left(\frac{a_{2}}{a_{3}}\right)^{n} & 1
\end{array}\right)
$$

where, in addition to replacing $U$ by $U^{(n)}$ we have expanded in the hierarchies to leading order. Notice for the case of half-integer $n, V_{L}$ depends on $U_{R}$ and vice versa.

\footnotetext{
${ }^{6}$ Also, for the simplicity of the argument we will in the following assume that the criteria on the sizes of the columns will coincide with the criteria on the sizes of $\left|U_{i \ell}\right|$.
} 
Open Access. This article is distributed under the terms of the Creative Commons Attribution License (CC-BY 4.0), which permits any use, distribution and reproduction in any medium, provided the original author(s) and source are credited.

\section{References}

[1] S. Antusch and V. Maurer, Running quark and lepton parameters at various scales, JHEP 11 (2013) 115 [arXiv: 1306.6879] [INSPIRE].

[2] C.D. Froggatt and H.B. Nielsen, Hierarchy of Quark Masses, Cabibbo Angles and CP-violation, Nucl. Phys. B 147 (1979) 277 [INSPIRE].

[3] N. Haba and H. Murayama, Anarchy and hierarchy, Phys. Rev. D 63 (2001) 053010 [hep-ph/0009174] [INSPIRE].

[4] J.A. Cabrer, G. von Gersdorff and M. Quirós, Flavor Phenomenology in General 5D Warped Spaces, JHEP 01 (2012) 033 [arXiv:1110.3324] [INSPIRE].

[5] E. Dudas, G. von Gersdorff, S. Pokorski and R. Ziegler, Linking Natural Supersymmetry to Flavour Physics, JHEP 01 (2014) 117 [arXiv: 1308.1090] [INSPIRE].

[6] T. Gherghetta and A. Pomarol, Bulk fields and supersymmetry in a slice of AdS, Nucl. Phys. B 586 (2000) 141 [hep-ph/0003129] [INSPIRE].

[7] S.J. Huber and Q. Shafi, Fermion masses, mixings and proton decay in a Randall-Sundrum model, Phys. Lett. B 498 (2001) 256 [hep-ph/0010195] [INSPIRE].

[8] R. Alonso, A. Carmona, B.M. Dillon, J.F. Kamenik, J. Martin Camalich and J. Zupan, A clockwork solution to the flavor puzzle, JHEP 10 (2018) 099 [arXiv:1807.09792] [INSPIRE].

[9] K.S. Babu, TASI Lectures on Flavor Physics, in proceedings of the Theoretical Advanced Study Institute in Elementary Particle Physics on The dawn of the LHC era (TASI 2008), Boulder, U.S.A., 2-27 June 2008, pp. 49-123 [https://doi.org/10.1142/9789812838360_0002] [arXiv: 0910.2948] [INSPIRE].

[10] R. Barbieri, G.R. Dvali and L.J. Hall, Predictions from a U(2) flavor symmetry in supersymmetric theories, Phys. Lett. B 377 (1996) 76 [hep-ph/9512388] [INSPIRE].

[11] R. Barbieri, L.J. Hall and A. Romanino, Consequences of a U(2) flavor symmetry, Phys. Lett. B 401 (1997) 47 [hep-ph/9702315] [INSPIRE].

[12] G.F. Giudice and M. McCullough, A Clockwork Theory, JHEP 02 (2017) 036 [arXiv: 1610.07962] [INSPIRE].

[13] G. von Gersdorff, Natural Fermion Hierarchies from Random Yukawa Couplings, JHEP 09 (2017) 094 [arXiv : 1705.05430] [INSPIRE].

[14] K.M. Patel, Clockwork mechanism for flavor hierarchies, Phys. Rev. D 96 (2017) 115013 [arXiv: 1711.05393] [INSPIRE]. 